\title{
Review Article \\ Relationship between Experimental Diet in Rats and Nonalcoholic Hepatic Disease: Review of Literature
}

\author{
Ayane A. Rodrigues, ${ }^{1}$ Raíssa S. B. Andrade, ${ }^{2}$ and Daniel F. P. Vasconcelos $\mathbb{D}^{1,2,3}$ \\ ${ }^{1}$ Postgraduate Program in Biomedical Sciences, Federal University of Piaui, Parnaiba, PI, Brazil \\ ${ }^{2}$ Postgraduate Program in Biotechnology, Federal University of Piaui, Parniba, PI, Brazil \\ ${ }^{3}$ Department of Biomedicine, Federal University of Piaui, Parnaiba, PI, Brazil \\ Correspondence should be addressed to Daniel F. P. Vasconcelos; vasconcelos@ufpi.edu.br
}

Received 30 May 2018; Revised 5 September 2018; Accepted 25 September 2018; Published 31 October 2018

Academic Editor: Heather Francis

Copyright (C) 2018 Ayane A. Rodrigues et al. This is an open access article distributed under the Creative Commons Attribution License, which permits unrestricted use, distribution, and reproduction in any medium, provided the original work is properly cited.

\begin{abstract}
Background. The pathophysiology of nonalcoholic fatty liver disease (NAFLD) is related to unhealthy lifestyles that combine sedentary lifestyle, hypercaloric diets, excessive saturated fats, refined carbohydrates, and high intake of fructose as a food additive to various processed products. Both the broader recognition of the disease and the additional efforts to elucidate the NAFLD pathogenesis have led to an increase in animal models in recent years. Objective. This review was performed to provide better understanding of the association between the NAFLD and animal models. Methods. The search in the literature occurred before May of 2018 in the PUBMED database. Results. Most studies investigating the influence of diet on liver fat content have been performed using a high-calorie diet that leads to a significant increase in fat content in the liver. Conclusion. The findings of this review show that diet is one of the factors that predisposes to the appearance of NAFLD and that the studies presented a wide variety of designs.
\end{abstract}

\section{Introduction}

The prevalence of predictive metabolic diseases, especially dyslipidemias and nonalcoholic fatty liver disease (NAFLD), has increased worldwide $[1,2]$. NAFLD and metabolic diseases associated with obesity, diabetes mellitus, and dyslipidemia are a growing public health problem both in the United States and worldwide [3]. The global population is affected by NAFLD with rates between $25 \%$ and $30 \%$ with higher prevalence in the Middle East and South America. In North America and Europe, a quarter of the adult population is affected by NAFLD [4]. It is a leading cause of liver transplantation and, by 2030, it is predicted to become one of the most common reasons for transplantation in the United States [5].

This pathology has drawn attention in recent years. Diagnosis and assessment of NAFLD can be performed based on physical examination, history of the disease, ultrasound examination, and hepatic imaging [6]. Although other noninvasive tests have been suggested, they must still be validated in large scales [1]. NAFLD presents an episode of variable hepatopathy of simple steatosis to nonalcoholic hepatitis (NASH), which is localized in the presence of hepatitis and inflammation, related to the death of hepatocytes; many patients progress to insufficient fibrosis/cirrhosis and hepatic failure [7]. The NAFLD is diagnosed histologically when $>5 \%$ of hepatocytes/field present lipid accumulation. That is the gold standard mean of diagnosis and should be considered in patients with NAFLD who present high risk to have fibrosis and steatohepatitis $[8,9]$.

The pathophysiology of NAFLD is associated with unhealthy lifestyles, which combine sedentary lifestyle, hypercaloric diets, excessive saturated fat, refined carbohydrates, and high fructose intake as a food additive to various processed products $[10,11]$.

Although the etiologies of dyslipidemias and NAFLD are complex and multifactorial, these diseases contain an important genetic component that is influenced by environmental factors and life habits, especially the food consumption profile $[2,12]$. 


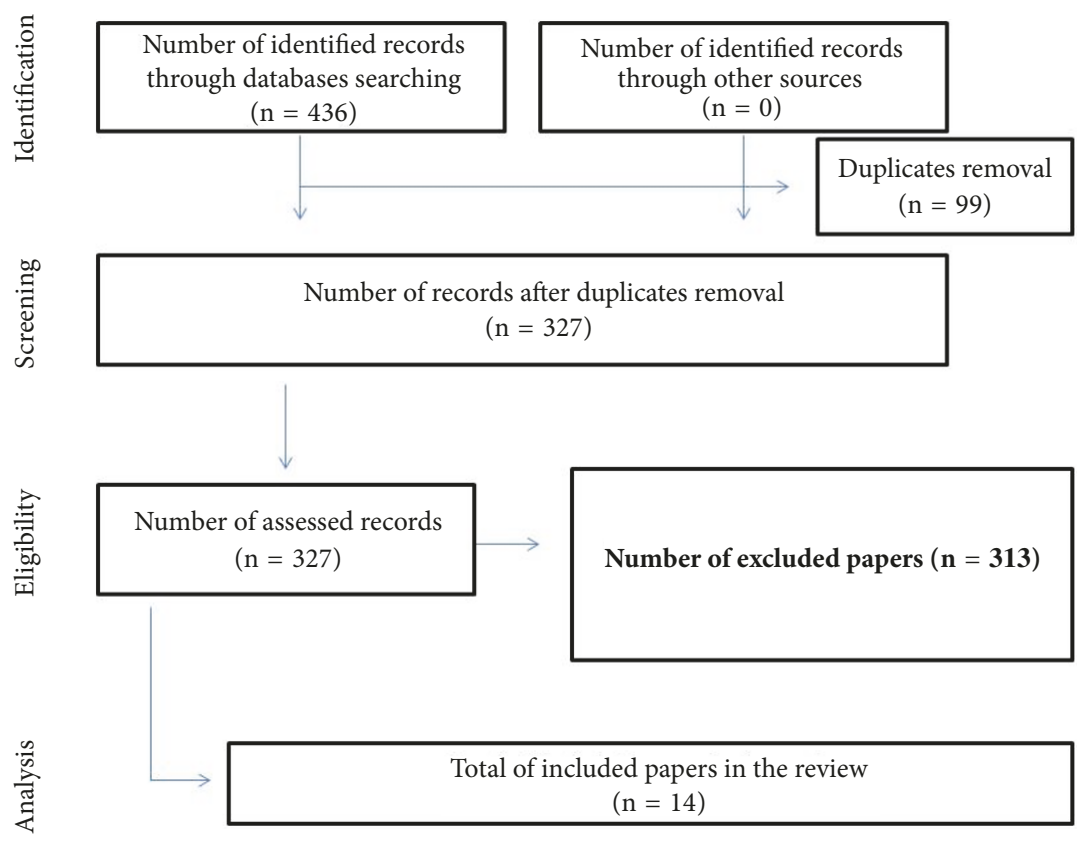

FIGURE 1: Flow diagram for identification, screening, eligibility, and analysis of studies included in the review.

Improved knowledge of the disease and its complementary data to elucidate the NAFLD pathogenesis have led to an increase in the animal models in recent years [13, 14].

Taking into account the increase in studies with NAFLD, the objective of this study was to investigate the different study designs that targeted the diet relationship with the appearance of NAFLD experimentally in rats.

\section{Materials and Methods}

This review was performed according to the PRISMA (Preferred Reporting Items for Systematic Reviews and MetaAnalyses) statement according to Moher et al., 2009 [15].

2.1. Eligibility Criteria. For the eligibility of publications, each title and abstract was read exhaustively to confirm whether they addressed the guiding question of this research and whether it would meet the inclusion and exclusion criteria established. Then, the same happened to the stage of inclusion of the articles. The selection of studies is shown in Figure 1. In the identification and screening, the following inclusion criteria were adopted: be available on the electronic address, be free of charge, be fully presented, and be disclosed in English, Portuguese, or Spanish. Investigations that did not present enough information about NAFLD were excluded. In addition, dissertations, theses, reports, news, letters to the editor, and scientific articles not fully available online and those that were repeated in the databases were excluded. Studies that used diet inducing alcoholic liver disease (ALD) were also excluded.

2.2. Search Strategy. The following keywords or descriptors were used in the Health Sciences Descriptors (DeCS) of the Virtual Health Library: "NAFLD", "diet", and "rat model”.
The search in literature was executed by two investigators in the databases: Cochrane Library, Google Scholar, and PubMed for studies published before 28 Mai 2018 and addressing the NAFLD associated with rat model. The following combined keywords were used to search the literature: ("NAFLD", "diet", and "rat model"). Figure 1 demonstrates the search strategy and details from the review.

\section{Results}

Through the search carried out in PUBMED, 436 articles were found, of which 89 articles were not related to the theme, 8 articles were repeated, 2 articles were not available, 2 articles were excluded due to the used diet, which induces alcoholic liver disease (ALD) and thus was not the focus of this review, and 313 were related to the subject, but they were testing other compounds. This way, 14 articles were analyzed (Figure 1). Regarding the periodical and language, they were all published in international journals with English language.

As for the articles discussing diet and the onset of NAFLD, 1 used the iron-fructose-enriched diet (Ackerman et al., 2005) [15]; 2 induced steatohepatitis through the diet deficient in methionine-choline (Kirsch et al., 2003; Wu et al., 2011) [16, 21]; 1 used the high-fat diet associated with sucrose (TorresVilalobos et al., 2015) [23]; 1 used the high-fat, fructose, and high-fat diets associated with fructose (Lee et al., 2015) [24]; 2 used the high-fat diet (Zou et al., 2006; McDonald, 2011) $[17,20] ; 2$ used a high-fat and a methionine-deficient diet (Xu et al., 2010; De Lima, 2008) [18, 19]; 1 used rich diet and fat and diet deficient in methionine and choline (Han et al., 2017) [28]; 4 reviewed experimental models that induce NAFLD (Kucera, Cervinkova, 2014; Eslamparast et al., 2017; Goossens, 2017; Mikhail et al., 2017) [22, 25-27]. The respective data are presented in Table 1. 


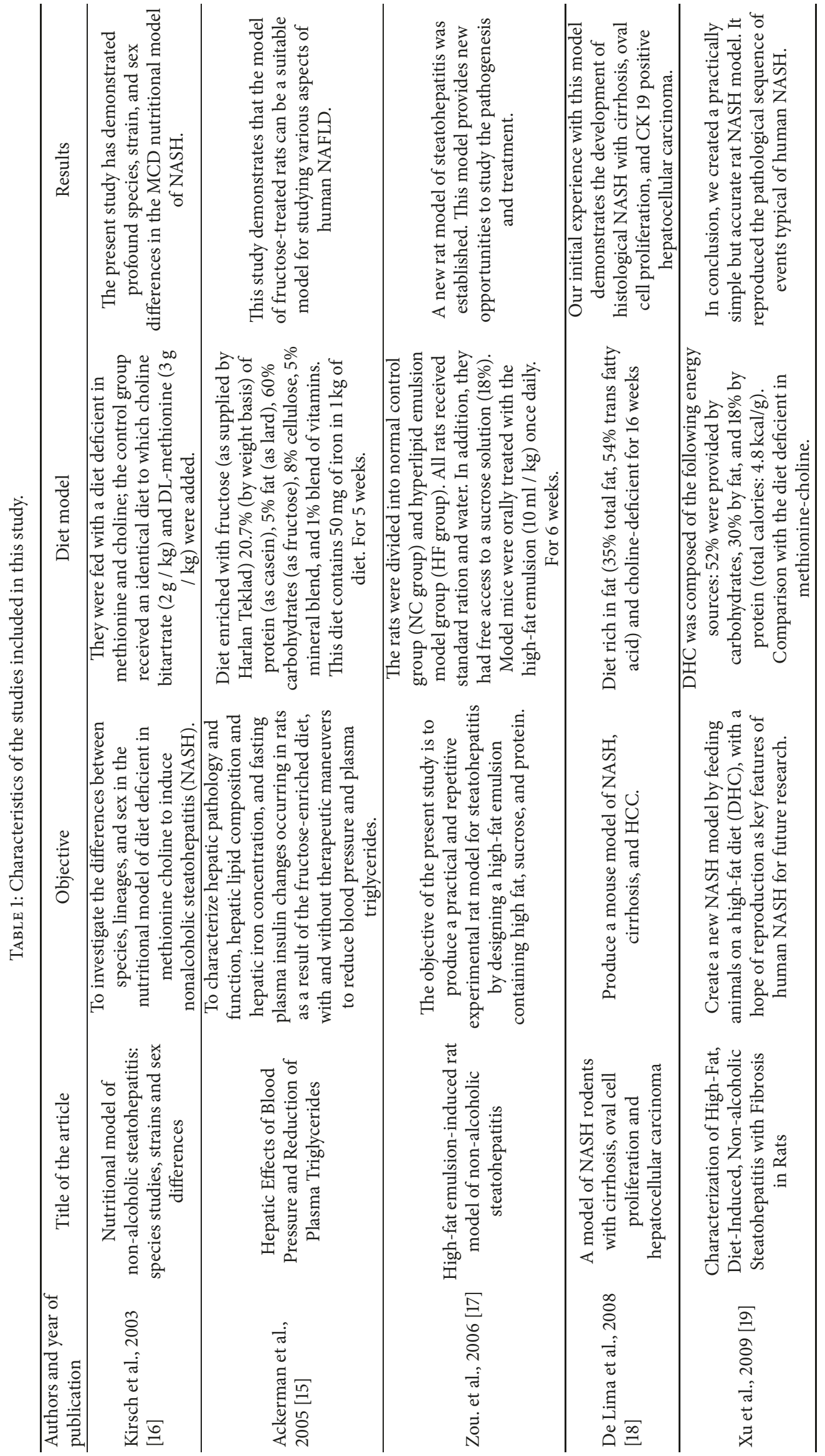




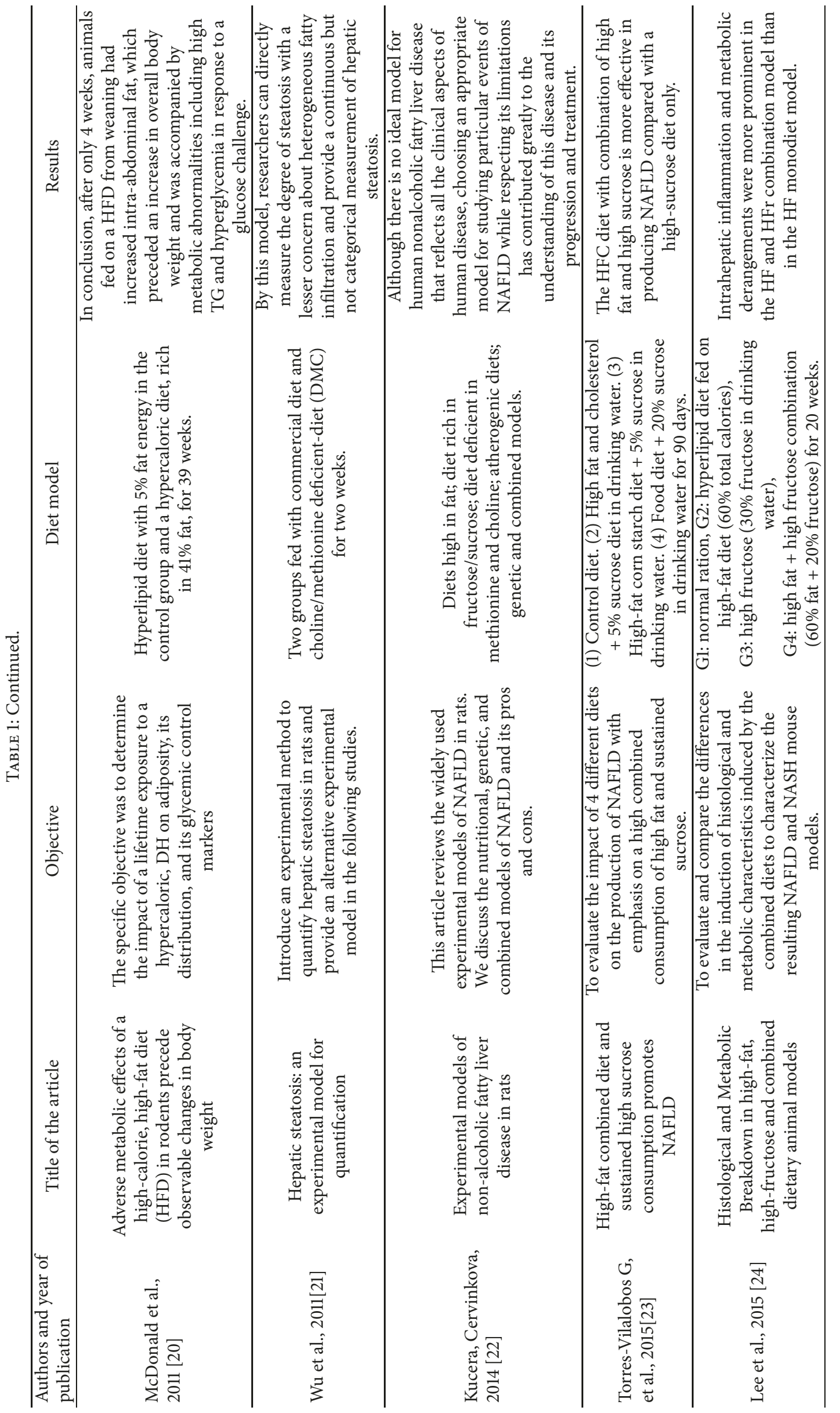




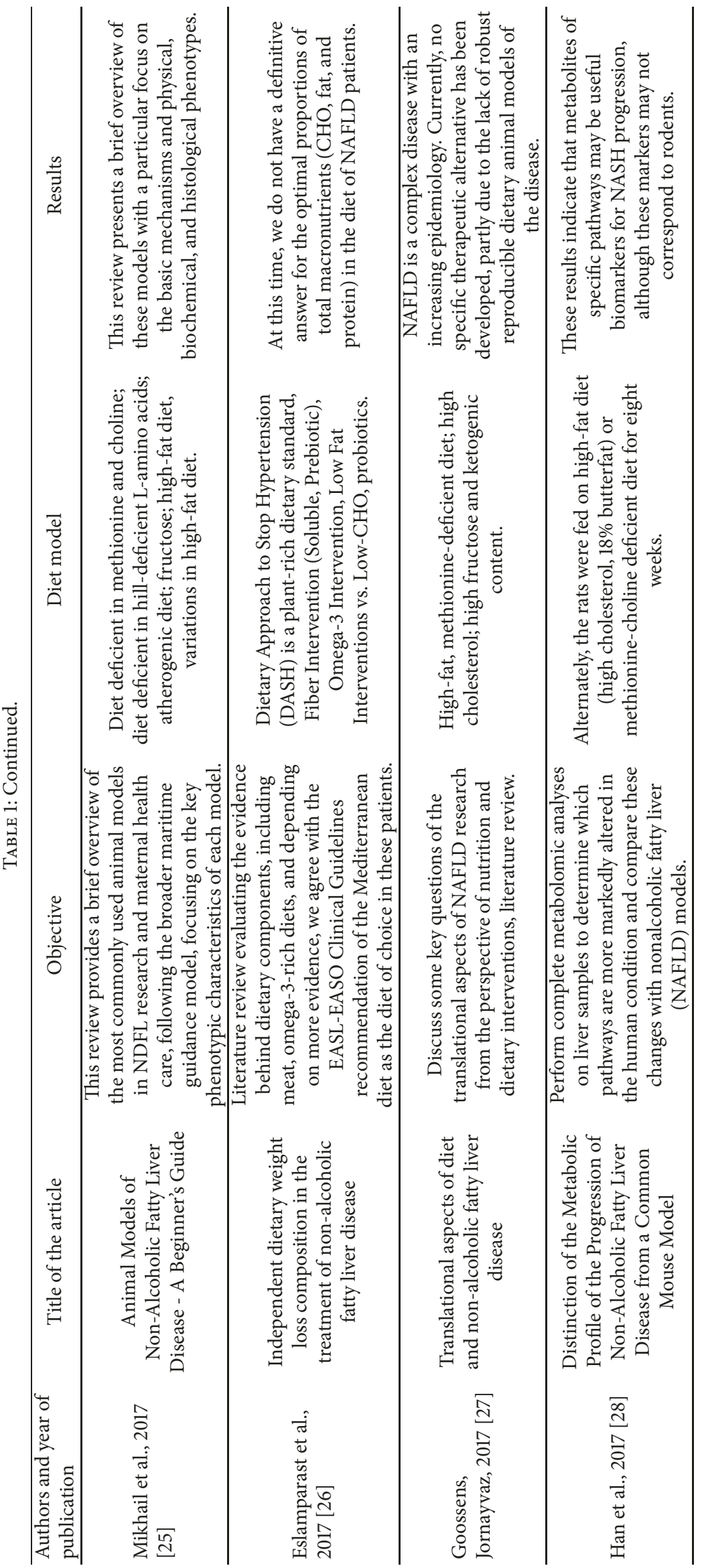


Dietary models are considered more similar to human metabolic diseases, but there are no standard composition and duration for these diets today: high-fat diets range from $30 \%$ to $60 \%$ fat content, including saturated fat, monounsaturated fatty acids, and polyunsaturated fatty acids, and last from a few days (short term) to more than a week (long term) [29]. In addition, theoretically composed diets of identical fat types can produce different results and data are often difficult to compare due to uncontrollable differences between primary fat sources and diet preparation [30].

It was observed that most articles found in the literature were related to the subject, but the studies used animal diet models to induce NAFLD in order to test compounds that interfere in the progression of the disease.

Ackerman et al. (2005) [15] demonstrated that the model of fructose-treated rats may be a suitable model for studying various aspects of human NAFLD, especially if it is associated with iron, since it generates an increase in liver fibrosis. This model contained $20.7 \%$ protein, $5 \%$ fat, $60 \%$ carbohydrate (fructose), $8 \%$ cellulose, $5 \%$ mineral blend, and $1 \%$ vitamin blend, combined with $50 \mathrm{mg}$ of iron for each $1 \mathrm{~kg}$ diet during 5-week period. The model of fructose-iron showed evidence of mild to moderate deposition of macrovesicular and microvesicular fat with minimal signs of perisinusoidal fibrosis in 3 of 12 rats.

De Lima et al. (2008) [18] proposed a diet rich in fat (35\% total fat, 54\% transfatty acid) and choline-deficient for 16 weeks. This model demonstrated the development of histological NASH with cirrhosis, oval cell proliferation, and CK 19 positive hepatocellular carcinoma. In 2009, Xu et al. [19] tested a high-fat diet in rats and serum levels of glucose, triglyceride, cholesterol, alanine aminotransferase (ALT), free fatty acids (FFA), insulin, and tumor necrosis factoralpha (TNF-alpha) were determined. Hepatic histology was also examined by H\&E stain. The liver weight and liver index increased within one month, when hepatic steatosis was also observed. By month 2, the body weight and epididymal fat weight started increasing, which was associated with increased serum levels of FFA, cholesterol, and TNF-alpha, as well as development of fatty liver. The serum ALT level increased from month 3 on. Steatohepatitis occurred after three months. Thus, this model is recommended for the study of NASH and its implications.

The study by Wu et al. (2011) [21] and later Han et al. (2017) [28] used a diet deficient in choline/methionine (methionine $0.15 \%$, choline $20 \mathrm{ppm}$, and $12 \%$ lipid component) for 2 weeks and concluded that researchers can directly measure the degree of steatosis with less concern about heterogeneous fatty infiltration and, moreover, provide a continuous, but not categorical, measure of hepatic steatosis.

The diet deficient in methionine and choline (MCD) in the study by Kirsch et al. (2003) [16] was composed of $2 \mathrm{~g} / \mathrm{kg}$ of choline and $3 \mathrm{~g} / \mathrm{kg}$ of methionine for 4 weeks. The research demonstrated profound differences between species, lineages, and sex in the nutritional model of MCD of NASH. From the groups studied, C57/BL6 male mice developed the histological characteristics that most resemble those observed in human NASH.

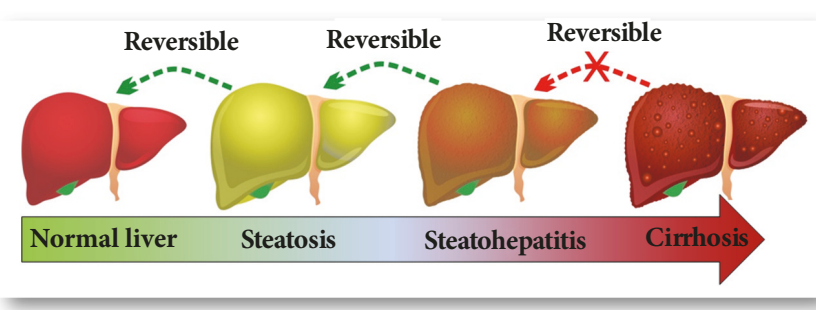

FIGURE 2: The progression of nonalcoholic fatty liver disease (NAFLD).

According to McDonald et al. (2011) [20], the highcalorie, high-fat diet with $41 \%$ fat energy was able to increase intra-abdominal fat, which preceded an increase in total body weight and was accompanied by metabolic abnormalities, including elevated triglyceride level and hyperglycemia in response to a glucose challenge.

Zou et al. (2006) [17] also stated that the hypercaloric diet model (77\% of fat energy, $14 \%$ of total milk powder, and 9\% of carbohydrates) provides new opportunities to study the pathogenesis and treatment of the metabolic syndrome associated with steatohepatitis, such as obesity, abnormal aminotransferase, hyperlipidemia, hyperinsulinemia, hyperglycemia, and insulin resistance. The progression of NAFLD also must be highlighted, which has potential to advance to steatohepatitis or even cirrhosis, as seen in Figure 2. It was demonstrated that the hypercaloric diet models showed the same metabolic profile observed in humans, such as increased levels of insulin, insulin resistance, hyperglycemia, hyperleptinemia, glucose intolerance, and increased levels of visceral white adipose tissue [17, 31].

The study by Kucera and Cervinkova [22] aimed to review several diets used to develop NAFLD and discuss nutritional, genetic, and combined models of NAFLD, as well as pros and cons. The choice of a suitable animal model for this disease, while respecting its limitations, may help to understand its complex pathogenesis and discover new therapeutic strategies.

In 2015, Torres-Vilalobos et al. [23] aimed to evaluate the impact of four different diets on the production of NAFLD with emphasis on high combined consumption of high fat and sustained sucrose. Their methodology used the following diets: control diet, high-fat, and cholesterol $+5 \%$ sucrose diet in drinking water, high-fat corn starch diet $+5 \%$ sucrose in drinking water, and food diet $+20 \%$ sucrose in drinking water for 90 days. They concluded that the high-fat + cholesterol diet with combination of high fat and high sucrose is more effective in producing NAFLD compared with a high-sucrose diet only.

Lee et al. (2015) [24] observed consequences of highfat $(\mathrm{HF})$ and high-fructose ( $\mathrm{HFr}$ ) diets in rats. Intrahepatic inflammation and metabolic derangements were more prominent in the HF and HFr combination model than in the HF monodiet model, which shows that a type of sugar aggravates the liver disease when associated with fat.

Eslamparast et al. (2017) [26] proposed in a review that poor dietary composition is an important factor in the 
progression of NAFLD and proposed that "high-quality healthy diet" improves hepatic steatosis and metabolic dysfunction in patients with NAFLD. They highlighted that most patients with NAFLD follow diets with overconsumption of simple carbohydrates and total and saturated fat, with reduced intake of dietary fiber and omega 3-rich foods.

Studies investigating the influence of diet on liver fat content were performed using a high-calorie diet that leads to a significant increase in liver fat content. DHC is well known to induce some metabolic disorders, and the consequences are completely dependent on the composition and duration of the diet $[32,33]$.

A broad view of the publications that study the diets used to simulate NAFLD in animals shows a break in nutritional, genetic, and combined diets. Goossens and Jornayvaz [27] describe the nutritional models of NAFLD that tried to mimic the metabolic disorders observed in the disease, as well as the histological alterations in the liver: hyperlipid diet, diet deficient in methionine and choline, high-cholesterol diet, high-fructose diet, ketogenic diet, and other models. Among these diets, Goossens and Jornayvaz [27] described the fatrich diet model mentioned, tested in male Sprague-Dawley rats by Lieber et al. [34] (71\% fat energy, 11\% carbohydrate, and $18 \%$ protein). They demonstrated the development of steatosis in three weeks associated with insulin resistance and increased fibrogenesis markers. This same study also suggests that the fructose-rich diet model associated or not with the high-fat model induces steatosis over a period of four to eight weeks. DHC is well known to induce some metabolic disorders and the consequences are completely dependent on the composition and duration of the diet [29].

According to Mikhail et al. [25], the chemical models are divided into streptozotocin, carbon tetrachloride, and diethylnitrosamine, while the genetic models are models of diabetes mellitus type 2 and models of atherosclerosis and hepatocellular carcinoma.

These models allow researchers to control, in vivo, genetic and environmental factors that may influence the development of the disease and its secondary complications [29], thus obtaining useful information about its management and treatment in humans.

\section{Conclusion}

In conclusion, the findings of this review show that the diet is one of the factors that predisposes to the appearance of NAFLD and that the studies presented a wide variety of designs, with the DHC being the most frequent diets in studies with experimental models of NAFLD. These reinforce that animal diet models are able to mimic the pathophysiological characteristics of NAFLD and are still widely used in research, mainly related to the testing of compounds that interfere with the progression of the disease.

\section{Data Availability}

The data used to support the findings of this study are included within the article.

\section{Conflicts of Interest}

The authors declare that they have no conflicts of interest.

\section{References}

[1] A. R. Araújo, N. Rosso, G. Bedogni, C. Tiribelli, and S. Bellentani, "Global epidemiology of non-alcoholic fatty liver disease/non-alcoholic steatohepatitis: What we need in the future," Liver International, vol. 38, pp. 47-51, 2018.

[2] R. W. Schwenk, H. Vogel, and A. Schürmann, "Genetic and epigenetic control of metabolic health," Molecular Metabolism, vol. 2, no. 4, pp. 337-347, 2013.

[3] T. Deng, C. J. Lyon, S. Bergin, M. A. Caligiuri, and W. A. Hsueh, "Obesity, inflammation, and cancer," Annual Review of Pathology: Mechanisms of Disease, vol. 11, pp. 421-449, 2016.

[4] Y. Fazel, A. B. Koenig, M. Sayiner, Z. D. Goodman, and Z. M. Younossi, "Epidemiology and natural history of non-alcoholic fatty liver disease," Metabolism - Clinical and Experimental, vol. 65, no. 8, pp. 1017-1025, 2016.

[5] J. Vizuete, A. Camero, M. Malakouti, K. Garapati, and J. Gutierrez, "Perspectives on Nonalcoholic Fatty Liver Disease: An Overview of Present and Future Therapies," Journal of Clinical and Translational Hepatology, vol. 5, no. 1, pp. 67-75, 2017.

[6] J. F. Cobbold, D. Patel, and S. D. Taylor-Robinson, "Assessment of inflammation and fibrosis in non-alcoholic fatty liver disease by imaging-based techniques," Journal of Gastroenterology and Hepatology, vol. 27, no. 8, pp. 1281-1292, 2012.

[7] X. Dou, S. Li, and L. Hu, "Glutathione disulfide sensitizes hepatocytes to TNFa-mediated cytotoxicity via IKK- $\beta$ S-glutathionylation: a potential mechanism underlying non-alcoholic fatty liver disease," Experimental \& Molecular Medicine, vol. 50, no. 7, 2018.

[8] S. Tandra, M. M. Yeh, E. M. Brunt et al., "Presence and significance of microvesicular steatosis in nonalcoholic fatty liver disease," Journal of Hepatology, vol. 55, no. 3, pp. 654-659, 2011.

[9] N. Chalasani, Z. Younossi, and J. E. Lavine, “The diagnosis and management of non-alcoholic fatty liver disease: practice guideline by the American Association for the Study of Liver Diseases, American College of Gastroenterology, and the American Gastroenterological Association," Hepatology, vol. 55, no. 6, pp. 2005-2023, 2012.

[10] L. Gerber, M. Otgonsuren, A. Mishra et al., "Non-alcoholic fatty liver disease (NAFLD) is associated with low level of physical activity: a population-based study," Alimentary Pharmacology \& Therapeutics, vol. 36, no. 8, pp. 772-781, 2012.

[11] B. A. Neuschwander-Tetri, "Non-alcoholic fatty liver disease," BMC Medicine, vol. 15, no. 1, article 45, 2017.

[12] J. K. C. Lau, X. Zhang, and J. Yu, "Animal models of nonalcoholic fatty liver disease: current perspectives and recent advances," The Journal of Pathology, vol. 241, no. 1, pp. 36-44, 2017.

[13] S. H. Ibrahim, P. Hirsova, H. Malhi, and G. J. Gores, "Animal models of nonalcoholic steatohepatitis: eat, delete, and inflame," Digestive Diseases and Sciences, vol. 61, no. 5, pp. 1325-1336, 2016.

[14] D. Moher, A. Liberati, J. Tetzlaff, and D. G. Altman, "ReprintPreferred Reporting items for systematic reviews and metaanalyses: the PRISMA statement," Physical Therapy in Sport, vol. 89, no. 9, pp. 873-880, 2009. 
[15] Z. Ackerman, M. Oron-Herman, M. Grozovski et al., "Fructoseinduced fatty liver disease: hepatic effects of blood pressure and plasma triglyceride reduction," Hypertension, vol. 45, no. 5, pp. 1012-1018, 2005.

[16] R. Kirsch, V. Clarkson, E. G. Shephard et al., "Rodent nutritional model of non-alcoholic steatohepatitis: Species, strain and sex difference studies," Journal of Gastroenterology and Hepatology, vol. 18, no. 11, pp. 1272-1282, 2003.

[17] Y. Zou, J. Li, C. Lu et al., "High-fat emulsion-induced rat model of nonalcoholic steatohepatitis," Life Sciences, vol. 79, no. 11, pp. 1100-1107, 2006.

[18] V. M. R. de Lima, C. P. M. S. Oliveira, V. A. F. Alves et al., "A rodent model of NASH with cirrhosis, oval cell proliferation and hepatocellular carcinoma," Journal of Hepatology, vol. 49, no. 6, pp. 1055-1061, 2008.

[19] Z. J. Xu, J. G. Fan, X. D. Ding, L. Qiao, and G. L. Wang, "Characterization of high-fat, diet-induced, non-alcoholic steatohepatitis with fibrosis in rats," Digestive Diseases and Sciences, vol. 55, no. 4, pp. 931-940, 2010.

[20] S. D. McDonald, E. Pesarchuk, A. Don-Wauchope, H. El Zimaity, and A. C. Holloway, "Adverse metabolic effects of a hypercaloric, high-fat diet in rodents precede observable changes in body weight," Nutrition Research, vol. 31, no. 9, pp. 707-714, 2011.

[21] T. Wu, L. Chen, S. Tsai, Y. Liaw, and B. Hwang, "Hepatic steatosis: An experimental model for quantification," Archives of Gerontology and Geriatrics, vol. 52, no. 2, pp. 164-166, 2011.

[22] O. Kucera and Z. Cervinkova, "Experimental models of nonalcoholic fatty liver disease in rats," World Journal of Gastroenterology, vol. 20, no. 26, pp. 8364-8376, 2014.

[23] G. Torres-Villalobos, N. Hamdan-Pérez, A. R. Tovar et al., "Combined high-fat diet and sustained high sucrose consumption promotes NAFLD in a murine model," Annals of Hepatology, vol. 14, no. 4, pp. 540-546, 2015.

[24] J. S. Lee, D. W. Jun, E. K. Kim, H. J. Jeon, H. H. Nam, and W. K. Saeed, "Histologic and Metabolic Derangement in High-Fat, High-Fructose, and Combination Diet Animal Models," The Scientific World Journal, vol. 2015, Article ID 306326, 9 pages, 2015.

[25] M. A. Van Herck, L. Vonghia, and S. M. Francque, "Animal Models of Nonalcoholic Fatty Liver Disease- A Starter's Guide," Nutrients, vol. 9, no. 10, 2017.

[26] T. Eslamparast, P. Tandon, and M. Raman, "Dietary composition independent of weight loss in the management of nonalcoholic fatty liver disease," Nutrients, vol. 9, no. 8, 2017.

[27] N. Goossens and F. R. Jornayvaz, "Translational aspects of diet and non-alcoholic fatty liver disease," Nutrients, vol. 9, no. 10, 2017.

[28] J. H. Han, A. L. Dzierlenga, Z. Lu et al., "Distinction of the metabolic profile of the progression of non-alcoholic fatty liver disease from a common mouse model," Obesity, vol. 6, pp. 10691076, 25.

[29] I. Lozano, R. V. der Werf, and W. Bietiger, "High-fructose and high-fat diet-induced disorders in rats: impact on diabetes risk, hepatic and vascular complications," Nutrition \& Metabolism, vol. 13, no. 15, 2016.

[30] R. Buettner, K. G. Parhofer, M. Woenckhaus et al., "Defining high-fat-diet rat models: metabolic and molecular effects of different fat types," Molecular Endocrinology, vol. 36, no. 3, pp. 485-501, 2006.
[31] L. Ghibaudi, J. Cook, C. Farley, M. van Heek, and J. J. Hwa, "Fat intake affects adiposity, comorbidity factors, and energy metabolism of Sprague-Dawley rats," Obesity Research, vol. 10, no. 9, pp. 956-963, 2002.

[32] A. Pocai, K. Morgan, C. Buettner, R. Gutierrez-Juarez, S. Obici, and L. Rossetti, "Central leptin acutely reverses diet-induced hepatic insulin resistance," Diabetes, vol. 54, no. 11, pp. 31823189, 2005.

[33] R. Buettner, J. Schölmerich, and L. C. Bollheimer, "High-fat diets: modeling the metabolic disorders of human obesity in rodents," Obesity, vol. 15, no. 4, pp. 798-808, 2007.

[34] C. S. Lieber, M. A. Leo, K. M. Mak et al., "Model of nonalcoholic steatohepatitis," American Journal of Clinical Nutrition, vol. 79, no. 3, pp. 502-509, 2004. 


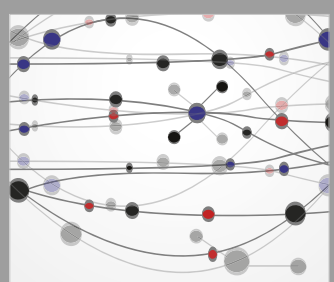

The Scientific World Journal
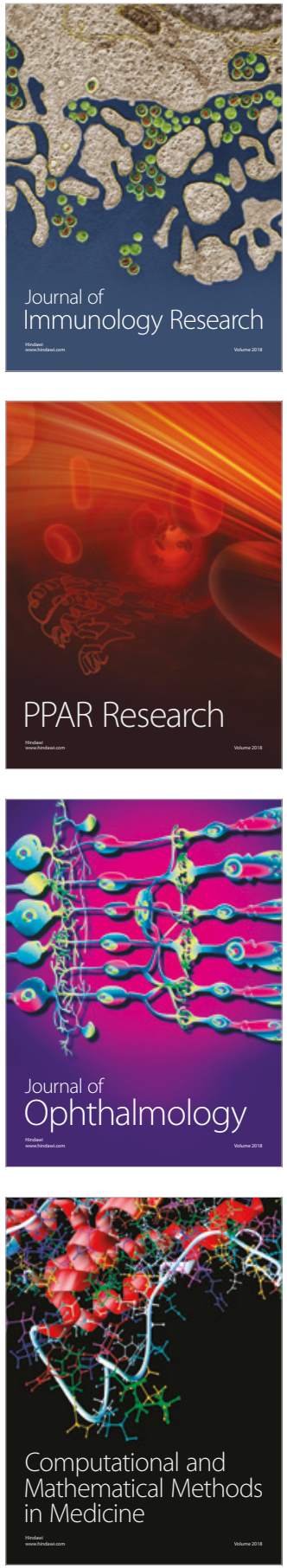

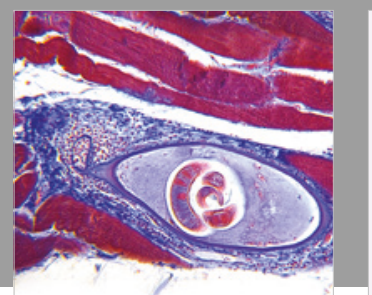

Gastroenterology Research and Practice

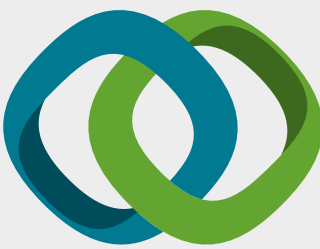

\section{Hindawi}

Submit your manuscripts at

www.hindawi.com
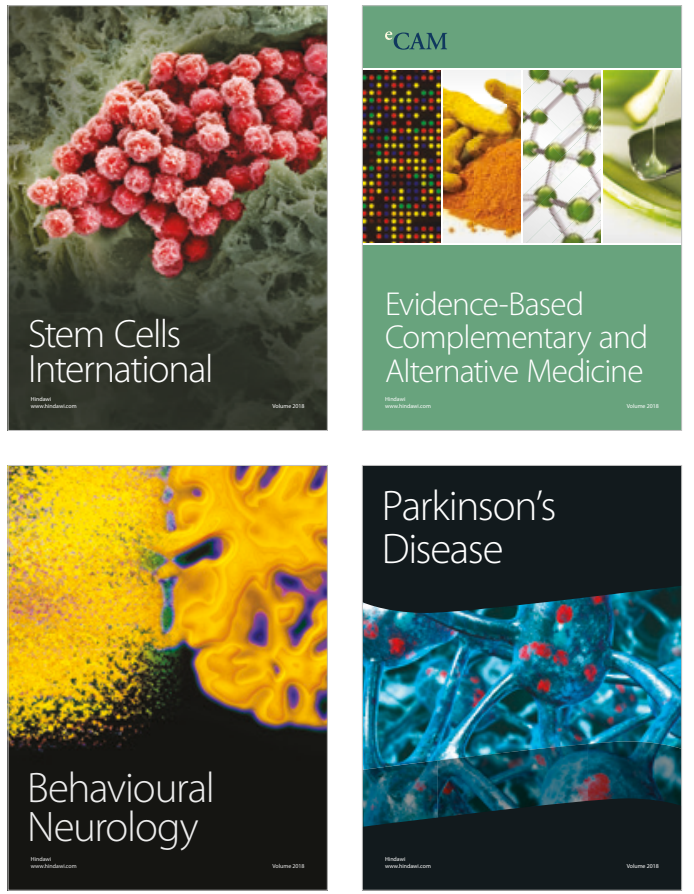

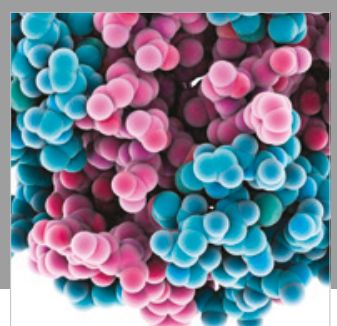

ournal of

Diabetes Research

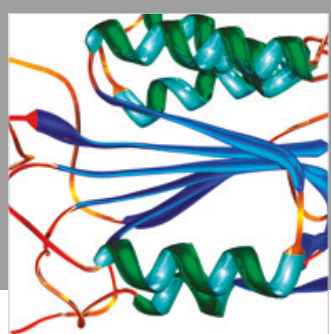

Disease Markers
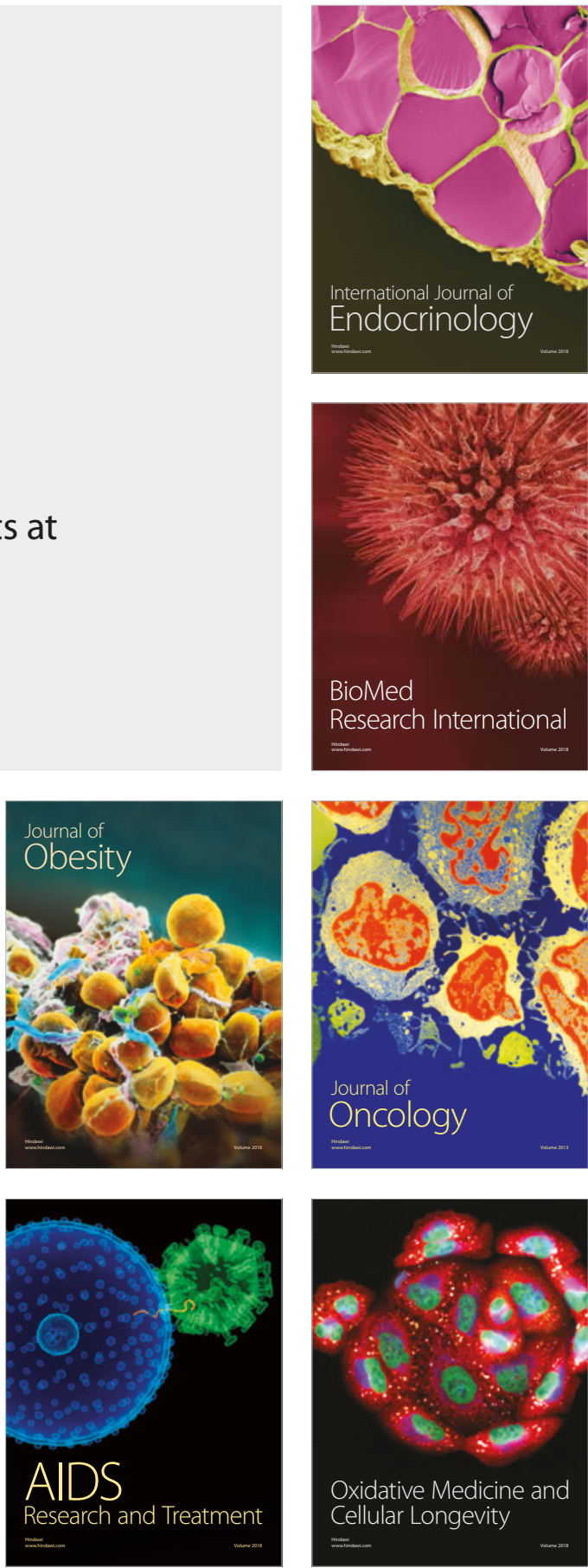